\title{
PRESENTACIÓN
}

Presentation

\section{LA HISTORIA DE LA EDUCACIÓN MÁS PERTINENTE PARA LA FORMACIÓN DE LOS DOCENTES: LA HISTORIA DEL CURRÍCULUM. NUEVAS PERSPECTIVAS, NUEVOS ENFOQUES, NUEVOS CONTENIDOS}

\section{Relevant History of Education for Teacher Education: Curriculum History. New Perspectives, New Approaches, New Contents}

\author{
Miguel Á. Pereyra \\ Universidad de Granada \\ Correo-e: mpereyra@ugr.es \\ Thomas S. Popkewitz \\ University of Wisconsi n-Madison. USA \\ Correo-e: popkewitz@education.wisc.edu
}

Recepción: I de septiembre de 202I. Envío a informantes: 5 de septiembre de 202 I.

Aceptación definitiva: 24 de septiembre de 202I

ste MONOGRÁfico está dedicado a Herbert M. Kliebard (I930-20I5) y Ba-
rry M. Franklin (I945-20I7), grandes scholars, colegas y amigos por mucho
tiempo, que con su saber, investigación, docencia y publicaciones impulsa-
ron la formación del campo de la historia del currículum

Agradecemos a nuestra colega y amiga Mónica Torres, de la Universidad de Málaga, la atenta lectura y comentarios de esta Presentación y a Luis Marín Romero, bibliotecario documentalista 


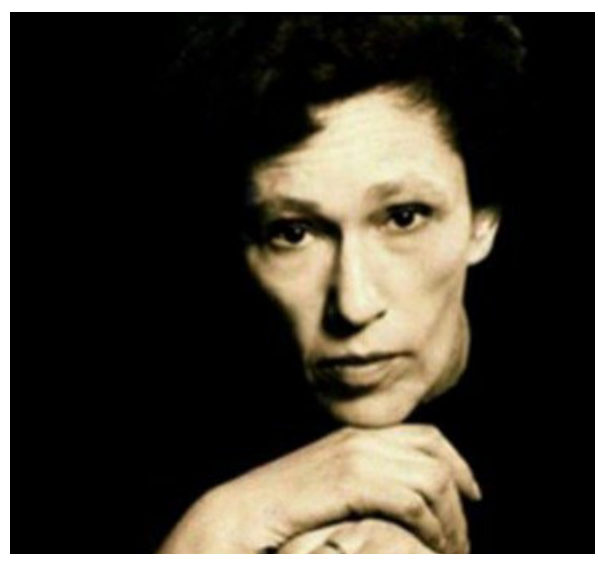

La historia de cualquier tipo connota, para mí, búsqueda, exploración y diseños cada vez más complejos... En este orden de cosas..., la importancia que tiene el estudio histórico para el docente reside en la posibilidad que ofrece para la configuración de grandes perspectivas, lo que permite a ese docente dar algún sentido al presente incipiente en el que se mueve. La investigación histórica consiste en la búsqueda de principios e ideas de las particularidades que componen la experiencia a medida que se amplía para incluir el pasado.

Haciéndose preguntas sobre las continuidades, las líneas de desarrollo, las relaciones entre ideas y acontecimientos, hechos y valores, y cambios económicos y sociales, el docente está en condiciones de perseguir significados, cuando no respuestas. Está en condiciones de orientarse en el universo continuo que se extiende mucho más allá de su situación personal. Está en condiciones de apropiarse del pasado haciéndolo parte de su propia situación vital, de las situaciones en las que debe tomar sus decisiones.

Maxine Greene (1917-20I4)

[«The Professional Significance of History of Education», History of Educational Quartely, 7(2) (1967), pp. 182-190]

Nuestro enfoque de la historia del currículum lo concebimos como un sistema de razón para comprender y explicar las huellas del pasado, pero también para hablar de cómo las estudiamos. Con los textos que producimos creamos paisajes que expresamos como historia cultural, como genealogía y como historia del presente; una orientación que puede proyectarse tanto en singular como en plural.

Desde hace más de dos décadas proponemos un debate en este sentido, del que nuestro libro Cultural History and Education. Critical essays on knowledge and schooling/Historia cultural y educación: ensayos críticos sobre conocimiento $y$ escolarización (T. S. Popkewitz, Barry M. Franklin y Miguel Á. Pereyra, 200I,

de la Biblioteca de la Facultad de Ciencias de la Educación de la Universidad de Granada, su apoyo técnico en el tratamiento e interpretación del impacto de algunas publicaciones, de las que aquí solo aparece reflejada una. 
traducido e impreso en español en 2003 por Ediciones Pomares de Barcelona) es un ejemplo relevante, por el impacto real que ha tenido desde que apareció. En el gráfico que reproducimos a continuación se proyecta el impacto que ha tenido la versión original en lengua inglesa desde su aparición, a partir del informe de citas que aporta la Web of Science.

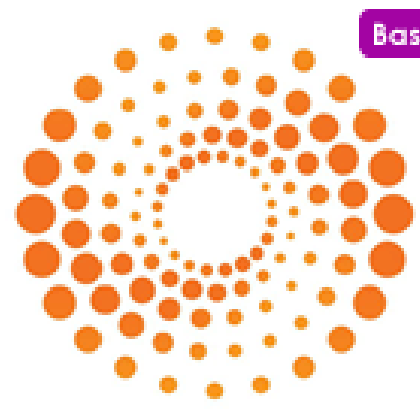

WEB OF SCIENCE'

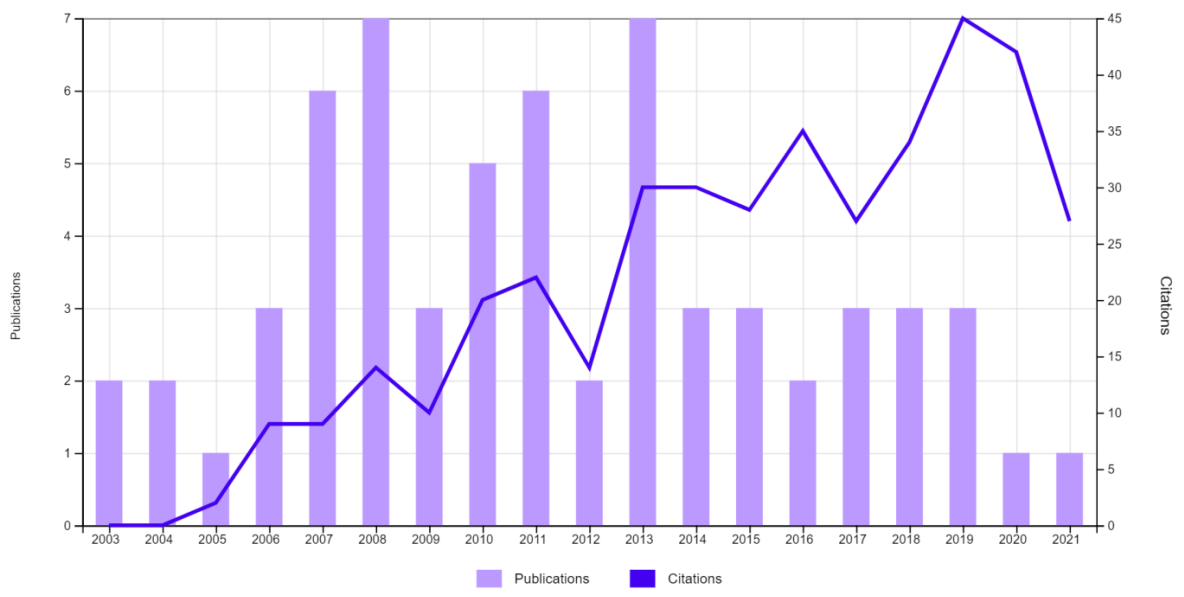

Informe de citas de Cultural History and Education: Critical Essays on Knowledge and Schooling, editado por T.S. Popkewitz, B. M. Franklin \& M. A. Pereyra (New York: RoutledgeFalmer, zoor y como ebook: Hoboken de Taylor and Francis, 2013).

Índice de impacto: 394 citas y 390 citas (sin autocitas)

En el gráfico i se muestra la relación por años entre el número total de citas del libro Cultural History and Education y el número de publicaciones en donde ha sido citado. Por ejemplo, en el año 2013, el libro fue citado 45 veces en 7 publicaciones diferentes. 
El monográfico de este volumen 40 de la prestigiosa y más antigua revista del campo de la historia de la educación en España tiene su procedencia intelectual como una continuación de nuestro libro que hemos compilado. Esta vez nuestro objetivo principal es poner en evidencia la importancia que han venido ganando la erudición y la investigación escrita orientada por un enfoque de historia cultural sobre un ámbito todavía menor que no ha tenido realmente el reconocimiento que se merece por el gremio de historiadores, ni en EE. UU. ni en España, en donde aparece ahora esta primera publicación de debate científico y académico. Sin embargo, vamos a sostener que el conocimiento que aporta el campo de la historia del currículum, de conocerse realmente en su concepción y enfoques puede tener una demanda y función en la formación del profesorado (en España, por ejemplo, cuyas universidades expiden todos los años miles de títulos de maestría universitaria para los que quieran optar a un puesto en la enseñanza secundaria pública o privada, ni ofrecen una materia específica sobre historia del currículum, con ejemplificaciones de las asignaturas o materias de la enseñanza secundaria, ni se incluye algún contenido de mínima solidez en otras materias teórico/histórico educativas).

La historia del currículum se desarrolló primeramente de forma más intensa en los EE. UU., sobre todo a partir de los últimos años de la década de 1960, y aún hoy genera nuevos tipos de preguntas, temas y enfoques de investigación. Este saber está en la actualidad muy asociado en los Estados Unidos, después de unos primeros tiempos unidos al protagonismo decisivo del Teachers College de la Universidad de Columbia, con la Universidad de Wisconsin-Madison (concretamente con su Department of Curriculum \& Instruction, que sigue siendo considerado después de más de treinta años de evaluaciones periódicas el primero de su clase, pero no el primero en crearse, que fue el del Teachers College en 1927). Desde las últimas décadas la historia del currículum se viene investigando y escribiendo también por scholars de Europa, América Latina, Asia y Australia, tal como se representa en este monográfico de Historia de la Educación.

\section{La llegada a España de la bistoria del currículum}

Del 23 al 26 de septiembre de 1996 se celebró en Granada el IX Coloquio de Historia de la Educación organizado por la Sociedad Española de Historia de la Educación (SEDHE) bajo el tema de El currículum: historia de una mediación social y cultural y al que asistieron $\mathrm{I} 69$ participantes, principalmente miembros de la Sociedad Española de Historia de la Educación (SEDHE), pero también algunos académicos de otros países y i8 estudiantes de los últimos años de licenciatura y del tercer ciclo de la Universidad de Granada (Sevilla, 19972². La conferencia inaugural Manuel Bartolomé Cossío la impartió el catedrático de la Universidad

Sevilla Merino, D.: «IX Coloquio de Historia de la Educación: El currículum: historia de una mediación social y cultural», Historia de la Educación, I6 (2013), pp. 68I-684. 
de Michigan-Flint Barry M. Franklin, con el título de Whatever happened to Curriculum History? The view from the United States of America, publicada en su original versión en inglés en los proceedings o actas del coloquio, entregadas a los participantes al inicio del coloquio. Estas las editaron los profesores del Departamento de Pedagogía más relacionados con los ámbitos académicos histórico, político y comparado, principales del nuevo campo, encabezados y dirigidos por la profesora Pilar Ballarín, entonces titular de Historia de la Educación, e impreso por Ediciones Osuna, en dos volúmenes, con el título o lema del Coloquio, siendo ella la propia editora de los dos volúmenes (véase El currículum: historia de una mediación social y cultural, Granada, Ediciones Osuna, 1996, 2 vols.).

Barry Franklin, reputado especialista en la historia del currículum estadounidense, en realidad uno de los creadores del nuevo campo, había publicado ya en la década de los setenta y ochenta varios trabajos y obras de referencia en este campo de investigación y en español, en particular en el primer volumen monográfico de los dos editados sobre Historia del Currículum, 295 y 296 de I991, de la Revista de Educación del Ministerio de Educación y Ciencia. La completa edición, con la selección de los estudios incluidos escritos por scholars principales del campo de Europa y América, habían sido compilados por Miguel Á. Pereyra, secretario/editor de la revista desde 1986 hasta finales de I992, cuando se incorporó a la Universidad de Granada, al obtener la cátedra de Educación Comparada que esta había convocado en 1991. Estos dos volúmenes suponen la fundación científica y académica del campo en España (y en general en el mundo de habla española, aunque hay que hacer notar que en Iberoamérica justamente en ese mismo tiempo aparecieron publicaciones que incluyen, en alguna de sus partes, reflexiones precisas y logradas sobre contenidos principales enfocados y relacionados con la historia del currículum, como es el caso de Sujetos, disciplina y currículum: en los orígenes del sistema educativo argentino (1885-1916), importante obra publicada por primera vez en 1990 por la conocida académica argentina Adriana Puiggrós).

La ponencia que con esmero preparó para la reunión científica de la SEDHE Barry Franklin, leyendo sobre nuestra historia contemporánea para comprender mejor quién fue y cuán grande fue Manuel Bartolomé Cossío, tenía como finalidad «examinar el estado actual de la historia del currículum, comparar la situación actual con el pasado del campo y plantear la importante pregunta de qué ha pasado con el historia del currículum» a lo largo de cuatro partes bien diferenciadas en las que profundiza en algunos de sus argumentos sobre el pasado y el presente de la historia del currículum, algunos de ellos, ya desarrollados en el artículo mencionado. Dado que esta conferencia no se publicó en español en ningún libro o revista, a continuación recogeremos una síntesis de las ideas más relevantes que nos servirán para introducir y contextualizar básicamente el campo de la historia del currículum.

Barry Franklin parte del trabajo pionero del que fue catedrático de Currículum y Enseñanza del Teachers College de la Universidad neoyorkina de Columbia, Arno Bellack (1969), que, aunque no consideraba la historia del currículum como un campo diferenciado de la historia de la educación [«i: History of curri- 
culum thought and practiced», Review of Educactional Research, 39(3) (1969), pp. 283-292], definió el contenido de la historia del currículum. Bellack señalaba, entre el desarrollo de las prácticas curriculares e instructivas, el crecimiento del currículum como campo de estudio, el estudio de los primeros pensadores significativos sobre el currículum y, finalmente, las recomendaciones y propuestas de los comités nacionales para estudiar el currículum. Terminó este famoso artículo diciendo que «La historia del pensamiento y la práctica del currículum no pueden separarse de la historia general de la educación estadounidense, que, a su vez, no puede separarse de la corriente más amplia de la historia cultural e intelectual» (p. 29I).

Esta definición de Arno Bellack va a permitir a Franklin trazar la evolución de este campo de investigación y diferenciar a historiadores del currículum de "primera generación» de las décadas de los sesenta, setenta y ochenta, que se centraron principalmente en el estudio de las ideas de varios intelectuales que pensaron sobre la educación y el currículum (John Dewey, Edward L. Thorndike, G. Stanley Hall, W. T. Harris y Charles Eliot), de aquellos educadores que tuvieron un papel más decisivo en la introducción del currículum como un área de estudio universitario, los denominados Curriculum Studies (Charles y Frank McMurry, Franklin Bobbitt, W. W. Charters, William Heard Kilpatrick, Hollis Caswell y Ralph Tyler) y que tuvieron un papel decisivo en organismos nacionales encargados de realizar propuestas para modificar los programas escolares a nivel local o nacional, algunos de ellos de gran impacto y que hoy siguen siendo referentes históricos de primera naturaleza. El estudio de las propuestas de cambio curricular de estos educadores, así como las ideas y movimientos pedagógicos fundamentales que defendieron (movimientos de eficiencia social, el progresivismo, el herbartianismo, por citar algunos), fueron abordados por los historiadores del currículum de «primera generación». Franklin apunta que, «por razones que no están del todo claras», estos primeros historiadores del currículum no reconocían la diferencia entre las recomendaciones sobre el currículum y la práctica curricular real.

Franklin apunta dos tendencias para caracterizar lo que ha sido la historia del currículum desde los años ochenta hasta aquel momento. La primera está relacionada con la ampliación de la agenda de investigación más allá del enfoque inicial de la evolución de las ideas sobre el currículum y las biografías de aquellos que lo defendieron. En este caso, Franklin desarrolla la contribución de la investigación de estudios de casos a la historia del currículum y enumera algunas de las que considera más relevantes: identifica el estudio de la reforma curricular en las escuelas públicas de Gary (Indiana) de W. Lynn McKinney e Ian Westbury de 1975 como pionero, al que suceden otros muchos durante las décadas de los 80 y los 90: el de Wayne Urban (198I) sobre la introducción de la formación profesional en la Atlanta de la era progresista, el de Barry Franklin (1982) sobre la reforma curricular en Minneapolis entre 1917 y 1950, el de Jeffrey Mirel y David Angus (1986) sobre los cambios en los planes de estudios de la educación secundaria en Detroit durante la Gran Depresión, el de David Labaree (1987) sobre la Central High School of Philadelphia y el de Herbert Kliebard (1996) sobre la construcción del concepto de currículum en el siglo XIX en Wisconsin. 
Estos estudios de casos han permitido realizar una revisión, una nueva interpretación y crítica de los primeros historiadores del currículum y, de manera más específica, de los postulados del movimiento de la eficiencia social y el cambio curricular. De manera más reciente el estudio de Popkewitz, Pereyra y Franklin (1996) trata de ofrecer unos «usos conceptualmente ricos y matizados de la teoría para explorar los problemas de la historia del currículum»3. Es decir, ofrece nuevas formas de pensar las ideas curriculares para mejorar la comprensión de la importancia de la teoría del currículum y su relación con la práctica escolar. En este orden de cosas, ya en 1983, en un libro de referencia importante, Kliebard y su querido y muy estimado intelectualmente student Barry Franklin terminaron definiendo el campo de la historia del currículum como «la propuesta académica de narrar, interpretar y finalmente comprender los procesos por los que los grupos sociales a lo largo del tiempo seleccionan, organizan y distribuyen conocimientos y creencias a través de las instituciones» (Kliebard, H. M. y Franklin, B. M.: «The course of the course of study: History of Curriculum», en Best, J. H. (comp.): Historical Inquiry in education. A research agenda, Washington, American Educational Research Association, 1983, p. 138).

Esta ampliación de la agenda de investigación afecta también a la historia de las disciplinas escolares. En este sentido, los historiadores del currículum estadounidenses han primado la historia intelectual o los estudios de caso para observar el desarrollo, la evolución o el cambio del currículum; en Gran Bretaña se centraron sobre todo en el análisis de la historia del currículum desde las disciplinas escolares, es decir, cómo los programas y la organización social de los conocimientos y la enseñanza varían a lo largo del tiempo en función de la configuración de los grupos sociales que detentan el poder académico y social. En este caso, las investigaciones realizadas de Ivor Goodson son las más destacadas. En los Estados Unidos, hay que destacar la única excepción que supone The formation of school subjects: The struggle for creating an American institution (1987), editado por Thomas S. Popkewitz, todo un referente reconocido en las revisiones principales y de importancia sobre la creación y desarrollo del campo de la historia del currículum en EE. UU. Es una compilación de los estudios escritos por relevantes scholars, todos norteamericanos, que entre otros temas pone el foco en cuestiones teóricas principales para el naciente campo, en este preciso tema de la formación de las asignaturas o materias escolares, como son la producción y el uso de las prácticas discursivas.

La segunda tendencia es el creciente interés de los académicos ajenos a los estudios curriculares, y especialmente de los historiadores de la educación, en esta empresa de investigación. Franklin argumenta que los primeros historiadores del currículum eran investigadores del currículum y que no será hasta I980 cuando los historiadores de la educación comiencen realmente a investigar sobre este ámbito. La incorporación de los historiadores de la educación al estudio de

Véase la primera parte de la obra Cultural History and Education (Nueva York: RoutledgeFalmer, 20oi), editada por T. S. Popkewitz, B. M. Franklin y M. Á. Pereyra. 
la historia del currículum ha permitido ir más allá de los estudios de las ideas y de las recomendaciones sobre el currículum propio de los estudios de "primera generación», para en definitiva ampliar y enriquecer el campo. Pero también la investigación sobre el currículum ha permitido que los investigadores con una orientación más teórica como los sociólogos o los estudiosos del currículum entren en un campo que ha sido «decididamente ateórico y descriptivo», de tal manera que la teoría crítica, la teoría del Estado o la teoría social postmoderna se han introducido en la agenda de investigación de la historia de la educación.

Franklin, en la parte final de su conferencia, ponderó positivamente los avances logrados por el nuevo campo científico en el poco tiempo pasado desde su surgimiento, hasta el punto de llegar a crear una asociación científica en 1977 (la Society for the Study of Curriculum History, que aún sigue reuniéndose anualmente en cada congreso de la AERA, y de la que aquí no podemos dar cuenta aunque próximamente sí lo haremos a partir de nuestra investigación, dada su importancia: fue promovida por profesores del campo del currículum a los que se les unieron, no muchos, historiadores de la educación, e inicialmente apoyó el más influyente de ellos, Lawrence Cremin). Barry Franklin concluyó en Granada diciendo, ante una audiencia que no mostró sinceramente demasiado interés por su ponencia, «... lo que ha sucedido con la historia del currículum en los Estados Unidos es que, al comienzo de su tercera década de existencia, se ha convertido en un lugar de investigación más estimulante, sólido y convincente de lo que la mayoría de los que construimos esta empresa podríamos haber imaginado» (pp. I9-20). Sin embargo, críticamente no se mostró, en ese 1996 y en la conferencia inaugural del Coloquio de Historia de la Educación de la SEDHE, del todo optimista ya que escribió, para concluir, acerca de una crítica y de una sugerencia para el desarrollo del nuevo campo.

La crítica no está basada en el escaso interés de los historiadores de la educación en el papel de la teoría y la generalización, sino más bien sobre las descripciones de acontecimientos o sobre actores individuales. Como sugerencia, introdujo en su ponencia una referencia al concepto del destacado historiador de la educación Carl Kaestle de historiar haciendo uso de la teoría «en un nivel teórico intermedio» (middle ground use of theory); es decir, de un «uso ecléctico o incidental de la teoría, no para predecir, sino para establecer modelos explicativos que permitan dar sentido a los datos y compararlos sobre todo a través de las fronteras nacionales» (p. 20). Argumentó y se posicionó finalmente sobre las ventajas que tendría la introducción de la teoría en la historia del currículum, pensando en que preocuparse por la cuestión de hacer uso de orientaciones de naturaleza teórica beneficiaría a la historia de la educación para explicar la cuestión histórica.

\section{Un nuevo campo científico y docente que debemos reconocer y desarrollar}

Este volumen se centra en una vertiente particular pero relevante de la historia del currículum que surge desde hace años del trabajo de Tom Popkewitz, pero 
en conversación intelectual mantenida en el tiempo con colegas como Ulf Lundgren, David Hamilton, Barry Franklin y Miguel Pereyra. Estas contribuciones divergen en varios sentidos de otros enfoques de trabajar la historia del currículum a través de las preocupaciones de cómo el conocimiento actúa para fabricar tipos de personas y, en la mayoría de los casos, en las tradiciones foucaultianas de genealogía e historización de la razón del currículum. Nuestra Presentación pretende esbozar algunas de las contribuciones de la historia del currículum al campo más general de la historia de la educación. El enfoque de este esbozo es, obviamente, limitado en su extensión con respecto a las diferentes perspectivas y orientaciones y contenidos de las contribuciones que forman el monográfico.

En los principios de este trabajo introductorio a este monográfico del vol. 40 de Historia de la Educación nos gustaría dejar escrita una preocupación principal. Se refiere al hecho de que pensamos que, propiamente dicho, las reflexiones a las que atienden esta Presentación no deberían ser englobadas con la etiqueta, que en varios sentidos puede ya catalogarse de obsoleta sobre todo en las ciencias sociales y por ende en la educación, de pensamiento postmoderno. El campo de la historia del currículum, aquí representado por las contribuciones de este monográfico, abarca hoy un espectro de estudios relacionados con lo poscolonial/decolonial, lo feminista, los estudios de ciencia y tecnología, las historias culturales y el nuevo materialismo, entre otros. Los estudios no conforman una homogeneidad teórica, sino que se orientan hacia lo que Wittgenstein llama «una familia de semejanza». En el centro de los estudios se encuentra la obra de Michel Foucault, y más recientemente Gilles Deleuze en las intersecciones que viene generando la scholarship contemporánea que se mueve a través de múltiples campos disciplinarios en las humanidades, la teoría social, la historia y la filosofía, pero inusualmente en la educación y rara vez en el campo académico de la historia anglonorteamericana de la educación como asimismo en otras culturas académicas de la educación.

A veces, esta literatura, como antes apuntábamos, se denomina erróneamente «posmoderna» para proporcionar una etiqueta categórica que determine tener un lugar dentro del conocimiento de la literatura científica. Esta etiqueta es poco útil desde el punto de vista intelectual y no es realmente reconocible en los estudios representados en este volumen. El ensamblaje de las literaturas que también antes aludíamos en este volumen de una revista veterana y especializada, no de divulgación, conlleva un tipo particular de «juego».

Utilizamos la palabra «juego» para expresar un método intelectual que no es reductivo ni que se concreta en meras aplicaciones de los conceptos o ideas, sino que es un «juego» productivo de ideas que tiene sus propias cualidades y distinciones. Los estudiosos e investigadores de este volumen, de diferentes generaciones aunque son más los de las últimas, trabajan en los intersticios de diferentes conjuntos de ideas para, por ejemplo, encontrar formas de pensar, métodos de estudio y estrategias de interpretación que planteen nuevos conjuntos de preguntas y cuestiones interpretativas para entender las condiciones de la Escuela moderna. 
Este «juego» es una cualidad intelectual que tampoco es un eclecticismo, sino una estrategia para desarrollar un estilo de razonamiento que permita moverse entre estos espacios diferentes, pero relacionados, de la erudición y el trabajo científico para precisamente «trabajar» sobre los eventos y documentos de la historia de la educación como artefactos culturales. A fin de cuentas, nuestras concepciones sobre la educación y la escolarización y sus prácticas y la investigación de las mismas deberían juzgarse por las cuestiones y preguntas que plantean, por los análisis a los que nos conducen y, por supuesto, por lo que terminamos aportando y reportando escribiendo sobre todo ello.

Cuando la inclusión de los contenidos de historia de la educación en los planes y programas de estudio para la formación del profesorado conoce en la actualidad un cuestionamiento mayor que el que ha estado presente en otras épocas desde comienzos del siglo xx, hay que hacer un esfuerzo por reflexionar sobre una situación de crisis de identidad de facto de la vieja disciplina académica. Tal crisis puede ser, en realidad, una crisis de conocimiento, por lo que habría que pensar en alternativas que ofrezcan dar más relevancia al campo de la historia del currículum, como argumentaremos al final de esta Presentación. Se puede plantear, por ejemplo, que tal relevancia reside en proporcionar una forma de repensar los principios que han impulsado las interpretaciones del pasado como un campo de estudio disciplinado o disciplinar.

Somos de la opinión de que el campo de la historia del currículum ha buscado -y se puede usar para ese fin- explorar los principios que rigen las formas de las preguntas históricas que nos hacemos, sus métodos y los significados asignados a sus fuentes y los lugares en los que se piensan; y también se pueden encontrar y explotar mejor para expresar y encarnar los juicios que hacemos, tal como los hacemos y escribimos sobre las narrativas de la historia.

Pensamos, en fin, en que las cualidades históricas sobre las que debe asentarse y desarrollarse el campo de la historia del currículum implican la intersección de conjuntos de teorías e ideas procedentes de las humanidades, la filosofía, la sociología política del conocimiento, los estudios sobre ciencia y tecnología y los estudios posfundamentales y posestructurales. Así, los estudios de este monográfico, en general, destacan la centralidad del conocimiento en el gobierno de la escuela y su relación con lo que es (im)posible hacer, pensar y decir.

Esta noción de conocimiento, entonces, no es el conjunto de ideas que tienen las personas y las instituciones presentes en la escuela. Se trata, más bien, de una preocupación por los principios sociales, culturales y políticos históricos, que son particulares y que se generan para producir distinciones, diferencias y divisiones sedimentadas como objetos de la organización y las prácticas institucionales de la escuela: el niño, su pedagogía y su currículum, y el maestro como objetos de planificación y gobierno de los modos de vida en la escuela.

En este enfoque que adoptaremos, una cuestión central es explorar cómo el objeto de la escolarización es la creación de tipos de personas que participan en la política de la modernidad. Pensemos, por ejemplo, que una de las características distintivas de la escuela moderna es la doble inscripción de la ciencia: como modo 
de interpretar y organizar la pedagogía y como cualidad psicológica de la mente que debe ser interiorizada en la racionalización del pensamiento de los niños. En este contexto, hay que pensar la cuestión principal y fundamental del poder en su conexión con el conocimiento y, en este sentido, es productivo en la creación de la voluntad de saber. El objeto, sin embargo, no es solo el comportamiento exterior, sino también la interioridad o, dicho metafóricamente, el alma de la persona.

En esta Presentación vamos a explorar estas clases de inscripciones de las que estamos hablando como una historia del presente. Sin embargo, las conexiones con el presente no tienen que ver con la forma en que se reproduce el pasado ni con la aplicación del presente para definir el pasado. Nuestro interés reside más bien en preguntarnos por las desiguales líneas históricas que se activan en el conocimiento contemporáneo o los sistemas de razón que ordenan el currículum, la enseñanza y la investigación sobre el niño, sujeto de la educación. También en este sentido, la «razón» de la ciencia la veremos como un «actor» histórico en el gobierno de la relación entre la pertenencia colectiva y la individualidad. Así concebida, la historia y el interés histórico quieren deshacer críticamente quimeras como las que suele exhibir la historia académica convencional empeñada en primar un realismo semiótico que conecta con el positivismo y el empirismo para romantizar el archivo, llegando a concebirse como la base metafórica en la que sustentar la cientificidad de la historia. Para tratar de desmantelar quimeras y mitos de esa naturaleza, nos vamos a mover entre múltiples literaturas disciplinarias.

\section{Una historia crítica del presente}

En un libro que acaba de aparecer, compilado por dos reconocidos scholars holandeses (Herman Paul, catedrático de Historia de las Humanidades, y Adriaan van Veldhuizen, profesor asistente de Filosofía de la Historia, ambos en la Universidad de Leiden), recogen y analizan la forma en que los académicos de las humanidades y las ciencias sociales de los siglos XIX y XX han venido debatiendo de forma más o menos intensa y apasionada los modos de pensar la historia y las traducciones y las filosofías de la historia arraigadas en los «regímenes de historicidad» que más de las veces se presentan como irreconciliables. En este orden de cosas, ellos sostienen que el historicismo es en sí mismo un concepto viajero o itinerante, por referirse a sus viajes a través de las disciplinas, los países y las divisiones confesionales religiosas, las mayoritarias protestantes y las católicas (Historicism. A Travelling Concept, 2021).

En esta Presentación argumentaremos que el historicismo es en esencia una visión ya clásica caracterizada por insistir en que la naturaleza de las cosas se encuentra en su historia y de ahí que este concepto se pueda usar como un prisma para una historia intelectual de cómo las personas se relacionan con su pasado. No obstante, debemos tener en cuenta el relativismo o el quietismo que ha solido acompañarle en sus viajes en diversos países y culturas, se haya producido hacia mediados del siglo xx un distanciamiento de él por parte de muchos intelectuales 
para abogar por un modo de erudición «más allá del historicismo» viajero. Este, efectivamente, terminó conduciéndonos a extremos muy relativistas en la posición asignada al pensamiento y en la justificación social, calificándolo muy bien Georg Simmel como historicismo determinista, que él trató de combatir en su filosofía de la historia.

Una crítica incluso más contundente la elaboró más tarde su compatriota Karl Löwith, quien en su búsqueda de un sentido y un fin para la historia encontró una vía intermedia, la de tratar de «historizar el historicismo», una frase que para él significaba ir más allá del historicismo sin relegarlo al basurero de la historia. Él creía que había que intentar deconstruir la conciencia histórica moderna, basada en una oposición entre el mundo histórico y el natural.

El objetivo de Löwith es reintegrar los acontecimientos históricos como partes de «la naturaleza de todos los seres», recuperar la «naturalidad de la historia». Para ello, apunta a una concepción de la historia como una «dialéctica del acontecer y del actuar»... Por un lado, la historia «sucede». Puede estar influenciada por la acción humana, pero, como dice, «lo que ocurre en la historia es siempre más y menos de lo que los humanos actuantes querían y pretendían. La dimensión del «acontecer» es especialmente importante, ya que significa que la historia no tiene una finalidad, ni un sentido, ni un sujeto... Con esta concepción, Löwith consigue que la historia en sentido clásico - relacionada con las acciones humanas- sigue siendo posible, pero el intento de captar la 'Historia' como un todo carece de sentido, ya que siempre está «sucediendo»y, por tanto, nunca está completa (Godefroy, B.: «Karl Löwith's historicization of historicism», en Paul, H.y Veldhuizen, A. van: Historicism. A Travelling Concept, Londres, 202I, pp. 205-207)

En nuestra concepción del historicismo hay conexiones con el pensamiento moderno de autores como los dos que acabamos de mencionar, pero en nuestro citado libro Historia cultural y educación (T. S. Popkewitz, B. M. Franklin y M. Á. Pereyra, 200I), nos posicionamos por considerar historicistas todas las ramas o diferenciaciones actuales del trabajo histórico, entendiendo por ello que el historicismo es una perspectiva general de la modernidad preocupada por comprender el pasado a través de las dimensiones de la temporalidad.

En nuestra compilación sosteníamos también que, para diferenciar entre el historicismo concreto de la historia social e intelectual más extendido por mucho tiempo y el historicismo de la historia cultural emergente en las últimas décadas, nos referimos al enfoque adoptado como bistorizante en el sentido de que contiene una «historia del presente» (p. 2o), que relacionamos con lo que antes decíamos de que las conexiones con el presente no tienen que ver con la forma en que se reproduce el pasado ni con la aplicación del presente para definir el pasado. Nuestro interés reside más bien en preguntarnos por las desiguales líneas históricas que se activan en el conocimiento contemporáneo o los sistemas de razón, un pensamiento más cercano a Löwith y su idea y necesidad de superar el historicismo. 
En este marco, la noción de la historia en general presente en las contribuciones que configuran este monográfico sobre la historia del currículum se plantea, por ende, como una cuestión histórica del presente, es decir, cómo le damos inteligibilidad al presente, dicho sintéticamente. De este modo, al leer los diferentes estudios que forman este monográfico podemos advertir cómo las prácticas históricas de la escolarización, cuando se piensan y analizan a través de su sistema de razón, no son propiamente producto de historias nacionales, ya que los flujos de ideas y realizaciones generados internacionalmente (o, más cercanos a los conceptos y expresiones de nuestro presente, transnacionalmente) son producto de dinámicas socioculturales y económicas que terminaron constituyendo e institucionalizando los diferentes sistemas educativos de los modernos Estados nación. En todos ellos encontramos, dicho brevemente, la inserción de visiones e ideas con impacto en el caso que nos ocupa de la historia del currículum.

En este campo de estudio que es la historia del currículum, también pensamos la historia como una forma de crítica. No se trata de encontrar fallos, sino de cortar lo que parece evidente para dar contingencias históricas. Este ejercicio intelectual, que es también un compromiso crítico y ético, lo hacemos, y lo logramos parcialmente, comparando el mundo de la escolarización con el conocimiento que tenemos de nuestro propio mundo y de nosotros mismos, lo que nos exige superar la producción de un conocimiento que se agota en sus formulaciones y referencias que terminan en abstracciones para, sobre todo, prestar atención al conocimiento que se produce y se da en la vida cotidiana y en las experiencias de la escolarización. Porque es este conocimiento concreto el que generan las personas a las que asignamos la función de categoría professional determinante sobre quienes son y pueden ser los maestros y los niños.

Complementariamente a lo que sostenemos sobre la relevancia de un enfoque de la historia del presente para orientar la práctica investigadora (y docente) de la historia del currículum, los estudios de este volumen plantean cuestiones relevantes sobre los principios filosóficos y sociales que subyacen a las prácticas del análisis histórico. Plantean cuestiones, por ejemplo, sobre el significado de la agencia, es decir, cómo pensar en la libertad y la ética de una manera diferente a la doxa actual.

También se plantean cuestiones sobre los métodos de los estudios históricos, particularmente sobre cómo estos se piensan desde el foco del recurrente protagonismo que se le da a la función del archivo. Pretendemos dimensionarlo mejor de cara, fundamentalmente, a la práctica de interrogación que suele asignársele como fundamento del trabajo del historiador. Los archivos suelen percibirse como eventos históricos y los documentos pueden verse, de hecho, como eventos, pero no para entenderlos, concebirlos y usarlos como textos fiables para expresar, configurar y articular ideas y programas, sino para preguntar cómo los textos enuncian conjuntos de ideas y distinciones que hacen posible lo que es visto y funciona o actúa como tema(s) de la escolarización. Por ejemplo, se trata de preguntar cómo es posible históricamente que los principios del currículum, la enseñanza y el aprendizaje actúen como herramientas de traducción en la for- 
mación de sujetos escolares como en los casos de la educación artística, científica y matemática.

Esta pregunta se hace posible a través de la exploración del currículum de las disciplinas o asignaturas escolares como una alquimia, una cuestión largamente tratada por Popkewitz en muchos trabajos y ahora sistematizada en su último libro (traducido recientemente), La impracticabilidad de la investigación práctica. Historia del tiempo presente de unas ciencias del cambio que nada cambian (Ediciones Octaedro de Barcelona; véase, en particular, el cap. 5).

Históricamente, los currículos de las asignaturas escolares han tenido poco que ver con el aprendizaje de prácticas disciplinares, es decir, los enunciados del currículum tienen menos que ver con el aprendizaje de la ciencia que con la producción de tipos de personas. El currículo escolar, como las alquimias medievales, supone trasladar un conocimiento desde un espacio social externo a la escuela; traducido y reelaborado en un conocimiento organizado por los principios pedagógicos y las psicologías del niño y del aprendizaje. La enseñanza de las ciencias, las matemáticas, el arte y la música a principios del siglo xx, por ejemplo, encarnaba tipos particulares de individualidades o clasificaciones determinadas que se relacionaban con los principios culturales y sociales que vinculaban las subjetividades con las imágenes y narrativas colectivas de la nación. Actualmente, esta alquimia en la política de las reformas educativas de los últimos años se expresa a través de la saturación de los discursos sobre las competencias clave para el siglo XXI sobre la fabricación de tipos de personas.

La alquimia de las materias escolares ha desarrollado un campo robusto en los estudios curriculares, que está fuertemente representado en este volumen. Las preocupaciones por el aprendizaje de las ciencias, las matemáticas y la educación cívica, por ejemplo, se producen en los espacios intermedios de las psicologías sociales y las psicologías del niño y el currículo escolar. El presente se convierte en un sitio anticipatorio para ordenar y desarrollar los modos de vida de los tipos de personas que los niños son y deben ser. Estos tipos de personas se denominan el niño que será un ciudadano activo y participativo, el aprendiz de por vida o el niño matemáticamente capaz.

Los documentos, entonces, ya no son datos, sino hechos dados a partir de los cuales los métodos de análisis interpretan los supuestos e implicaciones de lo que se dice. Por el contrario, los métodos deben estudiar las condiciones que hacen posibles sus reglas y normas internas sobre lo que se puede decir, pensar, convertir en memoria e institucionalizar. Los métodos tratan los documentos como acontecimientos para comprender cómo los sujetos históricos se hacen posibles como objetos de reflexión y acción.

La presencia de lo que podría pensarse como la cuestión empírica en las contribuciones del monográfico va entonces más allá de aportar datos para preguntarse antes por las reglas y normas de la razón archivadas en los textos sobre la escolarización. Se examinan históricamente las afirmaciones sobre la escolarización, el aprendizaje, los profesores y los niños para comprender los principios que ordenan y clasifican las objetivaciones en la escolarización. Así, más que tomar 
los documentos del archivo como origen para dar explicaciones, lo empírico es trabajar en la interioridad de sus espacios históricos en los que sus hechos se convierten en acontecimientos, archivados, por ejemplo, como índices de relaciones de poder en el gobierno de las personas y la vida social.

\section{Historicismo versus historización}

Recuperemos nuevamente el concepto de historicismo para prestar atención de nuevo a la metáfora del archivo, ahora en el sentido de que este es principal a la hora de dar significación para considerar los acontecimientos que hacen posibles los objetos de la vida social, más que como el depósito físico desde el que rastrear las condiciones que hacen posible el presente. Mientras que, paradójicamente, el historicismo organiza el pasado para hablar del futuro, pensamos y apostamos por la bistorización como un proyecto crítico para hacer frágil la aparente causalidad del presente. La historización se pregunta continuamente cómo hemos llegado al presente y cuáles son sus límites.

Es un método crítico para pensar en lo que somos y debemos ser y para pensar en el cambio. Como Foucault, pensamos que la crítica «es una cuestión de sacar ese pensamiento y tratar de cambiarlo: mostrar que las cosas no son tan evidentes como uno creía; ver que lo que se acepta como evidente ya no se aceptará como tal. Practicar la crítica es una cuestión de dificultar los gestos fáciles» (tal como le manifestó en 1981 a su biógrafo, el filósofo Didier Eribon, en una entrevista titulada Practicing criticism).

A fuer de sinceros, creemos que en la(s) práctica(s) de los historiadores de la educación no hemos llegado a hacer comprensible el conocimiento o razonamiento sobre quiénes son y deben ser las personas en la escolarización. Dicho de forma acaso más clara y concreta, aunque no nos guste leerlo, el tipo de razonamiento y el tipo de conocimiento que se busca y se logra, desde una práctica científica, no llega a alcanzarse, porque requiere comprender una gran cantidad de trabajo histórico, escrito sobre bases analíticas y no primando la erudición histórica tradicional, incluso cuando esta se deriva de fuentes consideradas desconocidas y sacadas a la luz a través de un trabajo archivístico que, sin duda, es arduo y puede ser meritorio... Más bien habría que decir, y reconocer, que este trabajo histórico se encuentra entre bastidores o fuera de la escena.

Hablamos entonces de historizar, cuyo objetivo es problematizar el tema objeto de investigación, que algunas veces se concibe y presenta como ahistórico o transhistórico (como sostuvo hace años Pierre Bourdieu, no existe un universal transhistórico de comunicación, sino que existen formas de comunicación socialmente instituidas favorecedoras de producir lo que puede ser universal y que están presentes en múltiples contextos con orígenes históricos y culturales no siempre convergentes).

Desde enfoques sustentados en la historia del presente que proponemos aquí, podríamos ampliar algo más nuestros argumentos y tomando el caso de una ins- 
titución histórica norteamericana de enseñanza superior sobresaliente en formación del profesorado, que ha sido creadora de campos científicos y disciplinares (y que, al mismo tiempo, ha impulsado investigaciones educativas importantes), como por ejemplo el desarrollo de los estudios curriculares y de la historia del currículum en particular, como es el caso del Teachers College de Nueva York. Aquí tenemos que, una vez lograda su afiliación con la Universidad de Columbia en $\mathrm{I} 898$, tras sus primeros inicios en I880 a partir de la creación de una asociación para la promoción de las artes domésticas industriales de las clases trabajadoras a través de programas educativos para ambos sexos, solo pudieron expandir sus objetivos de forma incluso casi sorprendente por los pocos años en que lo lograron, por la implicación desde el principio de filántropos como Grace H. Dodge, que no solo aportó dinero de su familia, sino que consiguió convencer a los Vanderbilt para que aportaran sumas importantes para la construcción del Teachers College y dotarlo de los medios materiales y profesionales; de forma que en unos diez años ya estaba preparada para convertirse en la escuela graduada profesional de educación, salud y psicología más grande de EE. UU. y se situó en el rango académico con las facultades de Derecho, Medicina y Ciencias Aplicadas. No tardó en llamársele «el West Point del trust educativo norteamericano» (hacia I950, el Teachers College había diplomado a un tercio de todos los presidentes y decanos de las instituciones norteamericanas de formación del profesorado y, al mismo tiempo, se consolidó como una de las instituciones con más influencia internacional en el campo de la educación).

Para una superación de las, en realidad, malas prácticas intelectuales anteriores proponemos la historización de la investigación, que nos lleve a descentrar al sujeto en el sentido foucaultiano. Para nosotros, la descentralización consiste en comprometer las complejas intersecciones que producen los principios que rigen lo que se piensa, se habla, se ve y se siente en la formación del sujeto. Esta descentralización del sujeto, con diferentes matices y focos, se pregunta cómo se producen las identidades de los sujetos en un tiempo desigual que no tiene un único origen. La historización que proponemos y que está presente en este volumen se pregunta cómo hemos llegado al presente y a sus límites. Y es un método para pensar en lo que somos y debemos ser y también en el cambio.

En concreto, la crítica consiste en sacar ese pensamiento y tratar de cambiarlo: mostrar que las cosas no son tan evidentes como uno creía; ver que lo que se acepta como evidente ya no se aceptará como tal. «Practicar la crítica es, en definitiva, una cuestión de dificultar los gestos fáciles», como manifestó Michel Foucault en la misma entrevista.

En conclusión, organizamos este monográfico para dar a conocer y poner en valor la investigación y erudición escrita dentro de la historia cultural. Lo que distinguió los primeros trabajos estadounidenses y europeos en este campo de los que se pensaron y escribieron en las décadas posteriores, dicho de forma general, fueron las historias sociales de los principios que rigen el currículum escolar y la pedagogía. Los estudios emblemáticos de Herbert Kliebard, alumno de Arno Bellack, y su alumno Barry Franklin fueron importantes para la reflexión y el 
flujo de ideas que organizaron. En Europa, investigaciones como las de UIf P. Lundgren, cuyo historicismo analítico y conceptual abordó las tradiciones cambiantes que organizaban la enseñanza y el aprendizaje, tuvieron resonancia en los trabajos de Basil Bernstein y David Hamilton, cuya historia social de la idea del currículum aportó importantes intervenciones sobre los principios sociales y culturales y sus cambios que organizaban las condiciones de la escuela formada como institución social.

4. Contenidos analíticos de las contribuciones del monográfico La bistoria de la educación más pertinente para la formación de los docentes: la bistoria del currículum. Nuevas perspectivas, nuevos enfoques, nuevos contenidos

A continuación, reseñamos brevemente los contenidos analíticos más relevantes de las contribuciones a este monográfico. Estas contribuciones están precedidas por dos estudios de investigadores españoles, el de los profesores de la Universidad de Cantabria Jesús Romero y su discípula Marta Estellés y el del catedrático de enseñanza secundaria de Historia Dr. Raimundo Cuesta, a quienes invitamos a aportar una exposición introductoria acerca de una evaluación analítica y crítica sobre la situación de la investigación sobre la historia del currículum en su corta historia de existencia en España.

Asimismo, en la sección de «Entrevista» se recoge una espléndida y elocuente entrevista con un docente que fue de enseñanza secundaria y luego un gran catedrático de Geografía Humana de la Universidad de Barcelona, en donde continúa como profesor emérito, Horacio Capel, pionero en la introducción en España de la investigación de la historia de las disciplinas escolares, sin ser él del campo de la pedagogía. Capel ha sido el director de una serie de sobresalientes tesis doctorales sobre la geografía enseñada en los institutos de bachillerato españoles. La entrevista la realizaron los profesores Xosé Manuel Souto González, de la Universidad de Valencia, y Francisco García Pérez, de la Universidad de Sevilla.

\section{Una breve historia de la educación matemática - Melissa Andrade-Molina}

¿Cómo se cuenta una historia? Lo que parece un objeto simple (la educación matemática), tiene múltiples dimensiones con diferentes formas de constituirse.

- También cómo se problematiza la forma en que la historia cuenta sus historias. Esta contribución aporta lo que aparece inicialmente como una historia conceptual de los orígenes de la educación matemática y argumenta sobre diferentes dimensiones de tiempo y espacios producidos continuamente por la evolución de una materia dentro de los campos de conocimiento y campos de prácticas. 
52 LA HISTORIA DE LA EDUCACIÓN MÁS PERTINENTE PARA LA FORMACIÓN DE LOS DOCENTES MIGUEL A. PEREYRA Y THOMAS S. POPKEWITZ

- Superar la historia de los orígenes o la visión de la historia como una evolución de una idea -el ideal platónico se traza como presente en la actualidad-.

- La historiografía tiene nociones multidimensionales y flexibles del tiempo, de ahí que se comporte como un rizoma que no se produce uniformemente en todos los países.

- El desafío es librar a la historia de la causalidad dada a través de sus nociones evolutivas, para pensar que entran en lo que constituye la historia de la educación matemática como campos de conocimiento, pero también como prácticas. Es una historia que no tiene un único origen.

Catolizar el cosmopolitismo: la estrategia retórica de la reforma curricular italiana de 2004 como reforma conservadora - Daniel Boccacci

- El estudio analiza el constructo de carácter cosmopolita promovido en la escolarización obligatoria italiana contemporánea a partir de Indicaciones Nacionales (2004), un documento curricular fundamental que ha proporcionado recursos para el sistema normativo que ha llegado hasta el presente escolar italiano.

- El autor destaca los significados históricos que constituyen las categorías de cristianismo y persona, que son la base del constructo cívico curricular contemporáneo, categorías fielmente tomadas de la pedagogía católica italiana desde la Segunda Guerra Mundial hasta hoy.

- Los documentos oficiales de la reforma curricular, así como el pensamiento y las acciones que se viven en aulas y escuelas, son considerados en el análisis aquí aportado como resultado de un proceso de producción de conocimiento y conductas que tiene origen dentro de un sistema amplio y dinámico de relaciones y prácticas sociales en el tiempo. En este sentido, el autor profundiza de manera transversal en el entrelazamiento histórico de las expectativas, decisiones y categorías de escuelas, pedagogos, la Iglesia católica, los partidos políticos, los empresarios...

- El análisis destaca especialmente el papel del componente persuasivo, no concebido como sistema simbólico-lingüístico, sino como una forma omnipresente y dinámica de prácticas curriculares que saturan el constructo persona, fuertemente metafísico, que se complementa con el razonamiento cosmopolita, concebido como un dispositivo universalizador que atraviesa legitimando, de manera conservadora, objetos del pasado y del presente de la política italiana y que converge en la prioridad otorgada a la enseñanza de la religión católica, disciplina capaz de orientar todo el currículo.

- Si bien el nuevo concordato entre la Iglesia y el Estado italiano estableció en 1986 la enseñanza no obligatoria de la religión católica, el cosmopolitismo personalizado derivado de las Indicaciones Nacionales de 2004 comporta un dispositivo para reactivar una forma de currículum concebido como 
MIGUEL A. PEREYRA Y THOMAS S. POPKEWITZ

«ordenanzas de conversión» (Popkewitz, 20Io), es decir, como transposición de la ética confesional católica a la escuela estatal, para el futuro sueño de una identidad patriótica nacional, republicana, mundial y competitiva.

Aceptar los límites históricos de la inclusión: cuando construir una mentalidad matemática no es suficiente - Jennifer Díaz

- Los objetivos de este estudio son analizar y problematizar cómo la educación matemática norteamericana crea imágenes del niño que se supone que encarna el tipo de mentalidad correcta para el aprendizaje de las matemáticas.

- Las matemáticas modelan, calculan y planifican un determinado tipo de futuro. La presunta utilidad de las matemáticas no tenía que ver con las matemáticas en sí mismas, sino que se concebían como una forma de racionalidad que podía aplicarse para modelar y planificar lo desconocido.

- Al entrar en el currículum a través del lenguaje y las reglas de la psicología, las prácticas pedagógicas organizan el currículum como formas particulares de ver, pensar y hablar sobre los niños. Conceptos tales como motivación, creatividad, interés, participación y las oportunidades en la vida inscriben las reglas de cómo los niños deben vivir con una mentalidad matemática.

- Este currículum forma un pensar comparativo servido para concebir y definir sobre quiénes contribuirían a la sociedad futura y quiénes no -los niños socialmente desfavorecidos-.

Quem tem medo de Euterpe? Para uma genealogia dos currículos de música em Portugal nos séculos XIX e XX - Ana Luisa Fernandes Paz

La autora aporta un análisis crítico-genealógico que interroga sobre quién fue el niño de las sucesivas reformas curriculares sobre las enseñanzas de la música en la educación infantil, primaria, secundaria y también en planes de estudio y los contenidos del conservatorio y de la Escuela Normal.

- En Portugal, en el desarrollo de la institucionalización de su sistema educativo a partir del segundo tercio del siglo XIX, se produjo un cambio relevante sobre lo que venía ocurriendo con la institución del conservatorio (en su origen este no estaba destinado a los músicos innatos, sino solo a asegurar que el raro talento no se desperdiciara por falta de condiciones favorables). De ahí que el desarrollo que se produjo no giró en torno a aprender a entender o producir música, sino a cantar en coro. Mucho más tarde, con la reforma producida en 1968, se sustituyó el canto coral por la educación musical y se buscó la manera de extender la formación profesional a un número cada vez mayor de personas. 
54 LA HISTORIA DE LA EDUCACIÓN MÁS PERTINENTE PARA LA FORMACIÓN DE LOS DOCENTES MIGUEL A. PEREYRA Y THOMAS S. POPKEWITZ

- En las diferentes etapas de la institucionalización de las enseñanzas de la música, la historización del niño del futuro muestra que existe un evidente paralelismo entre las diferentes modalidades de dicha educación que se desarrollan en una época, porque se sitúan en una racionalidad similar. Parece así posible confrontar el currículum musical mucho más allá de las diferenciaciones y ramificaciones institucionales, que no hacen más que operar como dispositivos de segmentación de personas, atendiendo a los mismos deseos y miedos sociales que el Estado centraliza y toma para sí.

Políticas contemporáneas para o ensino médio brasileiro: entre tradições disciplinares e o novo gerencialismo - André Vitor Fernandes dos Santos

- Esta contribución estudia la intersección de las políticas curriculares y las evaluaciones de las mismas en Brasil de acuerdo con los cambios de regímenes políticos de finales del siglo xx. Las nociones de competencias, expectativas de aprendizaje y dominio de habilidades en las políticas de evaluación encarnan reglas y normas sobre lo que cuenta como conocimiento.

- Diferentes formaciones discursivas dentro de las políticas curriculares contemporáneas producidas en el período que sucede al proceso de redemocratización de Brasil, desde la segunda mitad de la década de 1990, el final de la década de 2000, y el período entre 2014 y 2018.

- En 1990, con la redemocratización de Brasil, se creó el Sistema Brasileño de Evaluación de la Educación Básica y se identificaron los principios organizadores de lo que se caracterizó como la reforma de la escuela secundaria. En este contexto, la noción de competencia se concibió como un componente básico de la práctica discursiva de la reforma, mediando una relación entre el conocimiento escolar, considerado como tradicional, y las expectativas que surgen tanto del mundo del trabajo como de los espacios formativos posteriores a la escuela secundaria.

Diseñando gente, diseñando libertad. Los estudios de ciencia, tecnología y sociedad en las reformas educativas norteamericanas después de la Segunda Guerra Mundial - John P. Ivens

- El autor presta atención histórica a cómo se ensamblan los conocimientos particulares sobre los seres humanos, sus objetos y la democracia, y cómo los sistemas y el pensamiento sistémico funcionan como una tecnología de control social que diseña personas al tiempo que diseña su libertad.

- Ivens se centra en cómo los diferentes proyectos educativos traen de la teoría de los sistemas cibernéticos un conocimiento particular sobre la razón de los mismos que termina refiriéndose al diseño de los modos de vida. El lenguaje de las reformas entonces giraba sobre la libertad y la democracia, 
MIGUEL A. PEREYRA Y THOMAS S. POPKEWITZ

pero los usos de la teoría de los sistemas cibernéticos apoyaban y proponían, realmente, intervenciones basadas en tan recurrente teoría para regular los principios de pensar y actuar, así como los objetos de reflexión.

- En conclusión, la utilización de sistemas no busca mejorar la tecnología, sino mejorar a las personas, operando bajo una narrativa que simultáneamente libera a las personas en un orden de desarrollo emancipador cerrado, como fuerza social progresiva, pero creando mientras un sistema de control excluyente.

¿Un currículum acerca del juego libre? Un análisis multisituado de la bistoria de un currículum nacional de la educación infantil danesa - Maja Plum

- Esta contribución se ocupa menos de los actores y sus intenciones que de la forma en que se articulan y circulan a través de los tiempos las formas particulares de pensar sobre la naturaleza infantil y las maneras en que esta debe ser redimida. En lo metodológico, se pondera la búsqueda hermenéutica de sentido (desde un enfoque foucaultiano).

- La autora expone las formas particulares de pensar el juego, el niño y el jardín de infancia buceando en momentos particulares del tiempo que dan inteligibilidad al presente, y en aquellos en los que surgen rupturas en la forma de pensar el juego del niño y el jardín de infancia, al tiempo que se trazan líneas al presente.

- Cuando se está creando el ciudadano moderno, la infancia se piensa como espacio imaginado que conlleva una diferenciación entre edades y que, con el surgimiento de las nuevas disciplinas -como la psicología y la pedagogía- y los nuevos expertos, se quiere dar un cauce de su espiritualidad interior, para armonizarlo con las leyes eternas de la naturaleza.

- Jugar se concibe como algo muy distinto a las ideas de ociosidad; es un asunto de suma importancia que debe ser gobernado y prescrito meticulosamente para manejarlo de manera correcta.

- Estas prescripciones y el pensamiento de mediar el contenido del mundo a la naturaleza del niño a través de selecciones cuidadosas de las tareas conformaron un sistema de razón que hemos llegado a ver como central para el currículum y los estudios del currículum.

De la personalidad docente a la metodología de investigación: historizando la formación del profesorado basada en la investigación en Finlandia - Johanna Sitomaniemi-San

- Esta contribución analiza cómo las prácticas científicas conllevan racionalidades particulares para fabricar y gobernar al maestro/profesor. El análisis se fundamenta en la genealogía foucaultiana. 
- La autora estudia el tránsito de un ethos vocacional a un ethos de competencia profesional y el papel asignado al conocimiento científico. Investiga el papel de la alquimia de la pedagogía de la formación del profesorado y examina el pensamiento didáctico que se movilizó a partir de los años setenta.

- El modelo de formación del profesorado basado en la investigación supuso pasar de la influencia de la noción alemana de Bildung (formación) a la mirada norteamericana, y lo que se traslada de Haavio a Koskenniemi-del ethos vocacional al científico- es que hay que conocer la mente, o el alma, del profesor porque la personalidad de este es lo que hace únicos los procesos educativos.

- De este modo, la cientificidad de la formación del profesorado basada en la investigación funciona en consonancia con lo que parecen ser las ideas e ideales fijos de la educación, basados en el ethos Bildung, que es, a la vez, especulativo y vocacional, a pesar de sus contradicciones.

Historizando al niño abistórico del currículum portugués de educación artística en la enseñanza primaria. La educación artística en la escuela como forma de gobierno del alma del niño - Catarina S. Martins

- La autora estudia la particularidad de la educación artística que realmente no tiene que ver con las artes, sino que es una traducción de los principios psicológicos que organizan lo que el niño es y debe ser en la escolarización. Cuando se examina a través de estas lentes, la educación artística se relaciona con la actualización del ciudadano deseado para el futuro y no con las artes, concretándose así el proceso de alquimia en el que las materias escolares se usan y fundamentan con sus prácticas otros objetivos que no vemos ni reconocemos realmente cuando las enseñamos.

- Las asignaturas escolares, así como sus contenidos y la racionalidad que les dan sentido, no pueden verse entonces como derivadas de una esencia (lo que el niño es), sino de un conjunto de saberes y prácticas que dan razonabilidad a cómo pensamos lo que el niño es, y que tienen propósitos rectores.

- De los discursos curriculares sobre las artes en la educación primaria surge un niño que parece no tener historia: es un niño de un tipo determinado, que aprende de una manera determinada y que se desarrolla según una línea cronológica específica. Esta forma de pensar el niño tiene una historia, y esta historia se articula con prácticas de gobierno propias de una racionalidad moderna (sobre todo, por el desarrollo de la individualidad de cada sujeto). 
La resolución de problemas puede ser sometida a debate: fantasmagramas performativos de crisis en la reforma educativa STEM de Estados Unidos - Lei Zheng

- Las tecnociencias se denominan hoy en día en el medio escolar STEM (Science, Technology, Engineering \& Mathematics), una materia escolar cada vez más importante en las políticas transnacionales y las reformas educativas concebida como la representación de la innovación educativa del presente.

- Este estudio explora históricamente las formas particulares en que el currículum define las situaciones y los problemas que fueron imaginados y materializados a través de la educación STEM tendente a producir un tipo particular de niño solucionador de problemas.

- Se centra en cómo las multiplicidades de las tecnociencias recibieron un lenguaje unificado dentro de los currículos. Recurriendo a los estudios científicos poshumanistas de la performatividad, examina la historia del currículum como resultado de las dinámicas que se producen y funcionan como prácticas entretejidas en la organización del conocimiento, en lugar de considerar el currículum pasado como el origen de la organización de las prácticas escolares.

- La autora hace uso de la noción de fantasmagramas para pensar en el currículum de resolución de problemas como la inversión afectiva y la producción de imaginarios y sentimientos que animan los fenómenos de los niños que estudian las situaciones del mundo real y la resolución de problemas.

Un espejo para las personas - Disrupciones y aporias en documentos curriculares nacionales de Finlandia - Antti Saari y Tuomas Tervasmäki

Los autores estudian y analizan cómo se fabrican las representaciones de la ciudadanía en los discursos de los currículums nacionales en una época histórica en la que se percibe una dislocación; cuando la continuidad del orden político se ve amenazada.

- Los discursos curriculares finlandeses -los currículos nacionales, así como los textos académicos para la planificación, la implementación y la evaluación de los currículos desde la década de 1950 hasta la de 1970- construyeron una tesis cultural de una nación en la que los antiguos símbolos de unidad nacional -basados en nociones románticas de país, lengua y fe- se están evaporando rápidamente. Se consideraba que los profundos cambios políticos, tecnológicos e industriales requerían una adaptación y una capacidad de cooperación más generales que antes, lo que suponía una nueva orientación temporal o choque de futuro, cuyo objetivo era responder a las demandas futuras desconocidas en lugar de simplemente asegurar un sentido de continuidad histórica. 
- Después de la Segunda Guerra Mundial se invirtiieron las relaciones entre el Estado y el ciudadano, de tal forma que la persona dejó de estar sometida a la racionalidad y la agencia del Estado. En su lugar, el Estado obtuvo su legitimidad y la agencia de los intereses y deseos de sus ciudadanos.

\section{5. Últimas reflexiones}

La tradición de los estudios sobre el currículum que se explora en este volumen, en un país como España en el que su estudio ha sido muy tardío y, actualmente, la nueva reforma educativa de la enseñanza obligatoria recientemente aprobada por el Ministerio de Educación y Formación Profesional tiene su centro originario en los Estados Unidos, pero ha influido y se ha cruzado con el trabajo histórico que se realiza en Europa y también en países de América Latina, Asia y Australia.

Inés Dussel, del prestigioso DIE-CINVESTAV de México, al escribir recientemente sobre "The shifting boundaries of school subjects in contemporary curriculum reforms towards a post-disciplinary curriculum?», en una principalísima revista científica de la pedagogía académica germana, reflexiona sobre la relevancia del estudio de la historia de las disciplinas o asignaturas escolares, que es, precisamente, casi el único ámbito relacionado con la historia del currículum practicada en España, y que las dos contribuciones españolas de este monográfico de Jesús Romero y Marta Estellés y Raimundo Cuesta analizan y valoran juiciosamente. La relevancia de la historia de las disciplinas escolares acaso ahora es mayor, si cabe, que cuando no hace muchos años estas comenzaron a investigarse en centros de investigación y formación del profesorado porque, como expresa el subtítulo del artículo de Dussel, estamos hoy dando cauce a un «currículum posdiciplinar». De ahí que la investigación y la escritura de la historia de las disciplinas escolares se deba hacer usando e integrando la teoría curricular y no una historia esencialmente descriptiva.

Aquí es donde debemos volver a la relevancia que hoy tiene tratar de superar la crisis de identidad de la historia de la educación y su inveterada antipatía, cuando no rechazo explícito, al uso de enfoques conceptuales y analíticos para escribirla, a lo que en concreto se suele referirse como «la teoría».

Tal crisis de identidad es una crisis de conocimiento, como ya hemos aludido, resultado de una crisis con caracteres ontológicos, de ser y también de confianza en la historia de la educación como campo científico y de docencia. Maxine Greene, la gran filósofa existencialista de la educación, nos dejó algunas poderosas y muy provechosas reflexiones sobre la importancia de la historia de la educación en la formación profesional del profesorado, en unos momentos en que en EE. UU. se producía un vigoroso movimiento de reformas educativas del que emergió el vigoroso movimiento académico del revisionismo histórico en el campo de la educación, con debates de tendencias críticas tenidas como radicales y reacciones conservadoras entre los especialistas del campo, y todo ello con la 
sucesión de los movimientos y reivindicaciones sociales en las ciudades y en sus guetos, con las minorías y los inmigrantes, como los afroamericanos y el ascenso de la inmigración de hispanos o latinos... y con la guerra de Vietnam de terrible telón de fondo.

Precisamente los pensamientos que hemos transcrito al inicio de esta presentación a nuestro monográfico los escribió Maxine Greene, con la bella y a veces poética prosa que la caracterizaba, y los publicó en la revista principal de los historiadores de la educación norteamericanos estimulada por el revisionismo y el contexto sociopolítico que vivía su país entonces. La catedrática de Filosofía y Educación del Teachers College de la Universidad de Columbia afirmaba con rotundidad que era «evidente que tenemos que afrontar la difícil cuestión de la importancia de la disciplina en la formación del profesorado» porque, para ella, los historiadores de la educación se habían ocupado demasiado por su imagen pública en lugar de por su papel en la formación del profesorado, por adquirir el «estatus intelectual» de historiadores ante los tenidos por tales, pero lo cierto es que la American Historical Association en esos y en los siguientes años ni los conocía ni los reconocía.

Lawrence Cremin, factótum del campo de la historia de la educación desde joven, que llegaría a ser el séptimo presidente del Teachers College y a ganar un Premio Putlizer, cuando a mediados de la década de los cincuenta se produjo un amplio debate dentro del campo de la historia de la educación con la implicación de muchos historiadores de las principales universidades y facultades de educación, sentenció, en un informe relevante, que el declive de la disciplina, en un momento de crecimiento importante de centros universitarios de formación en todo el país, se debía a cuestiones referidas, sobre todo, al aumento de la demanda del «valor práctico en el currículum profesional y la afirmación de que la historia de la educación no servía a este valor» [Cremin, L. A.: "The role of the History of Education in the professional preparation of teachers. Part One», History of Education Journal, 7(I) (1955), p. 8].

Maxine Greene proponía, no muchos años después, en cambio, no preocuparse tanto por cuestiones así formuladas, sino por dedicarse a pensar y a producir, frente a la historia descriptiva tradicional en el campo de la historia de la educación, la historia explicativa, que debía suponer una conexión de la historia con otras ciencias sociales (pp. I82-183, del ensayo citado de 1967 al inicio de este ensayo nuestro). Esta conexión posibilitará que la historia de la educación trabaje sobre cuestiones sociales relevantes:

... la relevancia sigue siendo el quid de la cuestión en lo que respecta a la determinación de la importancia profesional; pero la relevancia, para mí, significa relevancia para el maestro y el profesor individual o el futuro maestro y profesor, no simplemente utilidad, y ciertamente no utilidad para mejorar el estatus de la profesión. (184) 
60 LA HISTORIA DE LA EDUCACIÓN MÁS PERTINENTE PARA LA FORMACIÓN DE LOS DOCENTES MIGUEL A. PEREYRA Y THOMAS S. POPKEWITZ

¿No es relevante y útil para la formación intelectual y para el futuro profesional de los docentes el conocimiento aportado por la historia del currículum? Creemos que la lectura atenta de las contribuciones, que las tenemos como investigaciones rigurosas e innovadoras, ayudará a clarificar la clase de cuestiones que presentamos. 